\title{
Accidentes por mordedura de canes atendidos durante el periodo 2017 - 2018 en un Hospital Nacional de Lima - Perú
}

\author{
Dog bite accidents treated between 2017 and 2018 in a National Hospital in Lima, Peru
}

Ivanna Door ${ }^{1}$, Daphne León ${ }^{1}$, Mario Mas ${ }^{2}$

RESUMEN

El objetivo del estudio fue describir las características epidemiológicas y clínicas de los accidentes por mordedura de canes atendidos en el Hospital "Sergio E. Bernales", ubicado en el distrito de Comas, Lima - Perú, durante los años 2017 y 2018. Para ello se elaboró una base de datos en el programa Microsoft Excel donde se transfirió la información de las historias clínicas correspondientes al 100\% de los pacientes atendidos por mordedura de canes en el periodo de estudio. Las variables consideradas en el estudio fueron: procedencia y características del paciente, condición y características del animal agresor, lugar del accidente, tipo de accidente, tipo de lesión, ubicación de la misma, atención de la herida y tratamiento realizado. La información se procesó haciendo uso del programa estadístico STATA 15.0 y se resumió mediante estadística descriptiva. Se recolectó información de 1598 fichas, predominando accidentados del sexo masculino $(56,4 \%)$ y del grupo etario de 30 a 59 años (30,4\%). Los accidentes más frecuentes fueron producidos por canes huidos (39\%). La mayoría correspondió a ataques sorpresivos (94\%), con lesiones leves $(54,8 \%$ ) y en miembros inferiores $(51,5 \%)$. La atención fue solicitada al día siguiente del accidente en la mayoría de los casos (53,6\%). Se espera que los resultados permitan mostrar el efecto de este tipo de accidentes sobre la salud humana y las autoridades correspondientes tomen las medidas preventivas a fin de atenuar sus efectos sobre la población.

PALABRAS CLAVE: Mordedura de canes, rabia, una salud, zoonosis

\section{SUMMARY}

The aim was to describe the epidemiological and clinical characteristics of dog bite accidents treated at Sergio E. Bernales Hospital, located in Comas district in Lima (Peru), between 2017 and 2018. A sheet was prepared in Excel to collect the information from the medical records, $100 \%$ of medical records of dog bites was transferred. The variables considered in the study were residence and characteristics of the patient, condition and characteristics of the dog, place of the accident, type of accident, type of and location of the injury, delay in seeking care for the injury and treatment received. Data was processed in STATA 15.0 and summarized using descriptive statistics. Information was collected from 1598 files, $56.4 \%$ of the victims were men and most of the victims were between 30 and 59 years old. Accidents caused by runaway stray dogs predominated (39\%). Most of the accidents were surprise attacks (94\%), with minor injuries (54.8\%), and in lower limbs (51.5\%). The results are expected to show the effect of this type of accident on human health in order to compel authorities to take preventive measures to alleviate their effects on the population.

KEY WORDS: Dog bite, rabies, one health, zoonoses

Laboratorio de Epidemiología y Salud Pública en Veterinaria, Facultad de Medicina Veterinaria y Zootecnia, Universidad Peruana Cayetano Heredia. Lima, Perú

2 Unidad Técnica de Sanidad Ambiental. Hospital "Sergio E. Bernales”. Comas, Lima, Perú 


\section{INTRODUCCIÓN}

Los accidentes por mordedura representan un riesgo para la salud de las personas debido a que es la principal vía de transmisión de la rabia y ocasionan infecciones bacterianas en las heridas, secuelas estéticas debido a las cicatrices, daños psicológicos, costos por el tratamiento de las heridas e incluso la muerte del agraviado en caso de accidentes muy graves (Morales et al., 2011; Arroyo et al., 2015; Talavera et al., 2018). Los niños suelen ser los más expuestos debido al estrecho lazo que tienen con los canes y por no ser capaces de identificar el comportamiento del animal que amenaza con morder, además de no contar con la fuerza física para poder defenderse (Morales et al., 2011; Arroyo et al., 2015; Novoa et al., 2017).

Los accidentes por mordedura están estrechamente relacionados con la agresividad de los animales, la cual se puede atribuir a una inadecuada crianza de estos, la genética, raza, socialización, etc. (Morales et al., 2011; Gallegos et al., 2018). Esta puede transmitir la rabia, enfermedad que ocasiona anualmente la muerte de 59 mil personas aproximadamente en el mundo (Organización Mundial de la Salud (OMS), 2018). Por ello, para controlar el riesgo de muerte por esta enfermedad, se debe cortar la transmisión a nivel del reservorio animal (Stokstad, 2017) y poner en práctica la triada preventiva: lavar la herida con agua y con jabón; identificar al animal agresor para su observación; acudir oportunamente a un centro de salud (Ministerio de Salud (MINSA), 2017).

En la evaluación médica que recibe una víctima por mordedura se debe tener en cuenta siete variables: tipo de contacto (mordida, arañazo/rasguño, lamedura, contacto con saliva), localización anatómica de la lesión, circunstancia en la que sucedió el accidente (si fue provocado o no), especie agresora, clasificación final del evento (exposición grave, leve o sin exposición a rabia), antecedente de vacunas del can agresor y antecedente de vacunas del agraviado (Saad y Martínez, 2014).

El distrito de Comas es el cuarto distrito con mayor población humana de Lima Norte (Instituto Nacional de Estadística e Informática (INEI), 2017) donde las condiciones de crianza que reciben los canes no serían las adecuadas ya que se observa canes deambulando en las calles. En este escenario la probabilidad de un accidente por mordedura de canes es mayor, sin que ello descarte los accidentes que puedan producirse en el hogar de los propios accidentados o en el domicilio de un tercero. Por ello, el objetivo del estudio fue describir las características epidemiológicas y clínicas de los accidentes por mordedura de canes atendidos en el Hospital "Sergio E. Bernales", ubicado en el distrito de Comas, Lima - Perú, durante los años 2017 y 2018.

\section{MATERIAL Y MÉTODOS}

El estudio de investigación descriptiva retrospectiva recolectó información en el Hospital "Sergio E. Bernales", ubicado en el distrito de Comas, Lima - Perú. El análisis de la información se realizó en la Facultad de Medicina Veterinaria y Zootecnia de la Universidad Peruana Cayetano Heredia. El estudio involucró la evaluación del total de fichas técnicas legibles de control por mordedura de canes registrados en el hospital durante los años 2017 y 2018.

La recolección de información se realizó en una base de datos en el programa Microsoft Excel donde se transfirió la información de las historias clínicas. Las variables consideradas fueron: distrito de procedencia del paciente (agrupadas por zonas según INEI, 2014), características del paciente (edad, sexo), características del animal agresor (edad, sexo, tamaño, estado vacunal), condición del animal mordedor (conocido, desconocido, huido, muerto), tipo de accidente (sorpresivo, provocado), tipo de lesión (leve, grave) y exposición de la herida (cubierta, descubierta), ubicación de la lesión (cara, cabeza, cuello, extremidad superior, extremidad inferior, tronco, pulpejos de los dedos de las manos y zona genital); días transcurridos hasta la atención post mordedura (día $0,1,2,3$ a 5 días, 6 a 9 días, más de 10 días) y tratamiento realizado (vacuna antirrábica).

El análisis de datos se realizó utilizando el programa STATA 15.0. Las variables cuantitativas se resumieron mediante estadística descriptiva utilizando medidas de tendencia central y de dispersión. En el caso de las variables cualitativas se utilizó frecuencias absolutas y relativas.

El estudio fue aprobado por el Comité Institucional de Ética de la Universidad Peruana Cayetano Heredia mediante constancia Nro. 252-12-19.

\section{RESULTADOS}

Se evaluó un total de 1598 fichas correspondientes a las atenciones de accidentes por mordeduras de canes registrados en la Unidad Técnica de Sanidad Ambiental del Hospital "Sergio E. Bernales", del distrito de Comas, Lima - Perú. Las personas afectadas 
eran principalmente de sexo masculino, del estrato etario de 30 a 59 años de edad y procedentes de la zona de Lima Norte (tabla 1).

La mayoría de los casos procedían de Lima Norte (1544). Estos se distribuyeron de la siguiente forma: 63,5\% (981) de Comas, 28,2\% (435) de Carabayllo, 4,1\% (63) de Los Olivos, 1,6\% (25) de San Martin de Porres, 1,4\% (21) de Puente Piedra, 1,1\% (17) de Independencia y $<0,1 \%$ correspondiente a un caso de Ancón y uno de Santa Rosa.

La edad de los afectados tuvo una media de 32.4 años, con mediana de 27 años y moda de 12 años. El valor mínimo y máximo de la edad fue 1 y 97 años, respectivamente. La mayoría de los accidentes se produjeron en el año 2018 y los meses que presentaron una mayor proporción de casos fueron enero $\mathrm{y}$ diciembre (tabla 2).

Tabla 1. Características de las personas que denunciaron accidentes por mordedura en el hospital "Sergio E. Bernales" en el periodo 2017 - 2018. Distrito de Comas - Perú ( $\mathrm{n}=1598)$.

\begin{tabular}{lcc}
\hline \multicolumn{1}{c}{ Variable } & n & \% \\
\hline Sexo & & \\
$\quad$ Masculino & 901 & 56,4 \\
$\quad$ Femenino & 697 & 43,6 \\
Estrato etario & & \\
1 a 4 & 129 & 8,1 \\
5 a 11 & 271 & 17,0 \\
12 a 14 & 112 & 7,0 \\
15 a 17 & 69 & 4,3 \\
18 a 29 & 265 & 16,6 \\
30 a 59 & 485 & 30,4 \\
>59 & 267 & 16,7 \\
Procedencia & & \\
Lima Norte & 1544 & 96,6 \\
Lima Centro & 11 & 0,7 \\
Lima Este & 4 & 0,3 \\
Lima Sur & 4 & 0,3 \\
Lima provincias & 1 & $<0,1$ \\
Callao Provincia & 3 & $<0,2$ \\
Otros Departamentos* & 4 & 0,3 \\
Sin información & 27 & 1,7 \\
*Juín (2), Ancash (1), Lambayeque (1). & \\
& &
\end{tabular}

Respecto al tipo y características de los accidentes por mordedura, estas fueron respondidas en diferente número para cada variable. Atendiendo a ello, el estudio mostró que predominaban los accidentes

Tabla 2. Distribución temporal de casos de mordedura de canes reportadas en el hospital "Sergio E. Bernales" en el periodo 2017 - 2018. Distrito de Comas - Perú $(\mathrm{n}=1598)$.

\begin{tabular}{lcc}
\hline \multicolumn{1}{r}{ Variable } & n & $\mathbf{\%}$ \\
\hline Año & & \\
2017 & 759 & 47,5 \\
2018 & 839 & 52,5 \\
Mes del año & & \\
Enero & 168 & 10,5 \\
Febrero & 176 & 11 \\
Marzo & 140 & 8,8 \\
Abril & 122 & 7,6 \\
Mayo & 118 & 7,4 \\
Junio & 96 & 6,0 \\
Julio & 101 & 6,3 \\
Agosto & 118 & 7,4 \\
Setiembre & 119 & 7,4 \\
Octubre & 123 & 7,7 \\
Noviembre & 152 & 9,5 \\
Diciembre & 165 & 10,3 \\
\hline
\end{tabular}

Tabla 3. Tipo y características de los accidentes por mordedura de can reportadas en el hospital "Sergio E. Bernales" en el periodo 2017 - 2018. Distrito de Comas - Perú.

\begin{tabular}{lcc}
\hline \multicolumn{1}{c}{ Variable } & $\mathbf{n}$ & $\mathbf{\%}$ \\
\hline Tipo de accidente $(\mathrm{n}=1582)$ & & \\
$\quad$ Sorpresivo & 1487 & 94,0 \\
$\quad$ Provocado & 95 & 6,0 \\
Tipo de lesión (n=1590) & & \\
$\quad$ Leve & 871 & 54,8 \\
$\quad$ Grave & 719 & 45,2 \\
Exposición de la herida $(\mathrm{n}=1436)$ & & \\
$\quad$ Cubierta & 639 & 44,5 \\
$\quad$ Descubierta & 797 & 55,5 \\
Atención de la vacuna $(\mathrm{n}=1406)$ & & \\
$\quad$ Sí & 568 & 40,4 \\
$\quad$ No & 838 & 59,6 \\
\hline
\end{tabular}


de tipo sorpresivos, de exposición leve, con heridas descubiertas y sin recomendación de vacunación (tabla 3).

En cuanto a la ubicación de las lesiones, las mordeduras predominaron las extremidades inferiores. En caso de dos lesiones, predominó la combinación de

Tabla 4. Localización anatómica de las mordeduras reportadas en el hospital "Sergio E. Bernales" en el periodo 2017 - 2018. Distrito de Comas - Perú.

\begin{tabular}{lcc}
\hline \multicolumn{1}{c}{ Localización Anatómica } & n & \% \\
\hline Mordedura únicas & & \\
Extremidad inferior & 811 & 51,5 \\
Extremidad superior & 428 & 27,2 \\
Cara & 128 & 8,1 \\
Tronco & 44 & 2,8 \\
Zona genital & 43 & 2,7 \\
Cabeza & 18 & 1,1 \\
Cuello & 2 & 0,1 \\
Mordedura doble & & \\
Extremidad superior / Extremidad & 38 & 2,4 \\
inferior & 10 & 0,6 \\
Extremidad superior / Zona genital & 10 & 0,6 \\
Extremidad inferior / Zona genital & 9 & 0,6 \\
Extremidad superior / Tronco & 7 & 0,4 \\
Cabeza / cara & 4 & 0,3 \\
Extremidad superior / Cara & 4 & 0,3 \\
Extremidad inferior / Tronco & 3 & 0,2 \\
Extremidad superior / Cabeza & 3 & 0,2 \\
Extremidad inferior / Cara & 2 & 0,1 \\
Tronco / Zona genital & 1 & 0,1 \\
Extremidad inferior / Cabeza & 1 & 0,1 \\
Cara / Cuello & 1 & 0,1 \\
Extremidad superior / Cuello & 1 & 0,1 \\
Extremidad superior / Pulpejo de & & \\
dedos & & 0,1 \\
Mordeduras triples & & 0,1 \\
Extremidad superior / Extremidad \\
inferior / Tronco & 3 & 0,2 \\
Extremidad superior / Cabeza / Cara & 1 & 0,1 \\
Extremidad superior / Cara / Tronco & 1 & 0,1 \\
Extremidad superior / Tronco /Zona & 1 & \\
genital & & \\
Extremidad inferior / Tronco / Zona \\
genital
\end{tabular}

miembros inferiores / miembros superiores. Cuando se trató de lesiones triples la combinación fue de miembros inferiores / miembros superiores / tronco (tabla 4). En cuanto a la atención post-accidente, predominó la solicitud de atención un día después del accidente (tabla 5).

Tabla 5. Días de atención post-mordedura de accidentes reportados en el hospital "Sergio E. Bernales" en el periodo 2017 - 2018. Distrito de Comas - Perú.

\begin{tabular}{|c|c|c|}
\hline Días post mordedura & $\mathbf{n}$ & $\%$ \\
\hline 0 & 205 & 12,8 \\
\hline 1 & 857 & 53,6 \\
\hline 2 & 275 & 17,2 \\
\hline 3 a 5 & 185 & 11,6 \\
\hline 6 a 10 & 58 & 3,6 \\
\hline Más de 10 & 18 & 1,1 \\
\hline Total & 1598 & 100,0 \\
\hline
\end{tabular}

Tabla 6. Características del can agresor de accidentes reportados en el hospital "Sergio E. Bernales" en el periodo $2017-2018$.

\begin{tabular}{lcc}
\hline \multicolumn{1}{c}{ Variable } & n & $\mathbf{\%}$ \\
\hline Sexo (n=320) & & \\
Macho & 238 & 74,4 \\
Hembra & 82 & 25,6 \\
Edad (años) (n=290) & & \\
$<1$ & 30 & 10,3 \\
1 a 2 & 91 & 31,4 \\
3 a 5 & 109 & 37,6 \\
Mas 5 & 60 & 20,7 \\
Tamaño (n=325) & & \\
Pequeño & 47 & 14,5 \\
Mediano & 138 & 42,5 \\
Grande & 140 & 43,1 \\
Vacunado (n=213) & & \\
Sí & 145 & 68,1 \\
No & 17 & 8 \\
No sabe & 51 & 23,9 \\
Condición del can mordedor (n=757) & & \\
Conocido & 255 & 33,7 \\
Desconocido & 183 & 24,2 \\
Huido & 295 & 39 \\
Muerto & 24 & 3,2 \\
\hline
\end{tabular}


En cuanto a las características del can mordedor, las preguntas para cada variable fueron respondidas en diferente número. Predominaron animales mordedores de sexo macho, tamaño grande y mediano, y la mayoría habría estado vacunada contra la rabia. La condición del can mordedor que predominó fue el de animales huidos, seguido de los animales conocidos (tabla 6). La edad de los canes mordedores se obtuvo en 184 animales, obteniéndose una media de 3.7 años, con mediana y moda de 3 años y valor mínimo y máximo de 0.17 y 14 años, respectivamente.

\section{DISCUSIÓN}

El estudio recuperó 1598 fichas correspondientes a accidentes por mordedura de canes en el período 2017-2018. La mayoría de casos pertenecieron al distrito de Comas, pero también se registraron casos provenientes de otros distritos de Lima Norte y otras provincias y departamentos del Perú, probablemente debido a que el accidente se produjo en tránsito por el área de influencia del Hospital, al que se recurrió para la atención del accidente.

La mayoría de registros de accidentes por mordedura corresponden a personas de sexo masculino, lo que suele estar asociado a las diferentes conductas de los varones, tanto adultos como niños, que pueden resultar desafiantes para los canes. Una serie de estudios entre los que se incluyen los de Schvartzman y Pacín (2005), Morales et al. (2011), Cubillos (2014), Chacma (2016), Gallegos et al. (2018) y Talavera et al. (2018), encuentran que la población más afectada correspondía a personas del sexo masculino. Se estima que, los varones pueden ser más rudos en sus actos, tener una conducta menos temerosa y despreocupada, además de interactuar más con los canes durante el juego; comportamiento que podría estar asociado a una mayor exposición al riesgo de una mordedura de can (Palacio et al., 2005).

Los accidentes por mordedura predominaron en el estrato de 30-59 años, población adulta que representa la población económicamente activa, quienes con su actividad laboral dinamizan la economía y genera ingresos para sus familias. Es probable que en el traslado a sus centros laborales se encuentren mayor tiempo en la vía pública, exponiéndose a mordeduras de canes. Eizaguirre (2016) señala que los accidentes por mordedura en personas adultas que trabajan, repercute en la economía de las familias ya que se debe pagar un tratamiento para curar la herida y puede haber pérdida de horas de trabajo de la persona mordida.

Sin embargo, los resultados del estudio difieren de los encontrados por otros autores, quienes señalan que los niños suelen ser los más afectados, debido a la susceptibilidad de su condición física ya que tienen menos fuerza y no saben distinguir los signos previos de un animal preparado para atacar (OMS, 2018). En ese sentido, un estudio a base de encuestas realizado por Talavera et al. (2018) en Puno y Madre de Dios, encontró que los grupos etarios de 6 a 10 y de 11 a 15 años resultaron ser los más afectados.

La mayoría de los accidentados buscaron atención médica el día siguiente de haber sido mordidos. Esto guarda relación con que la mayoría de accidentes fueron clasificados como leves, por lo que el accidentado le resta importancia a la atención de este tipo de heridas inmediatamente y en algunos casos no se reportan, con lo que se contribuye a la sub notificación y perjudica la valoración real de la cantidad de personas que sufren accidentes por mordedura. Según Schvartzman y Pacín (2005), existe un sub registro de este tipo de accidentes y que los mismos se reportan principalmente cuando los agraviados son niños o cuando se presentan lesiones complicadas en el caso de adultos. Sin embargo, la notificación de la mordedura debe ser realizarse siempre debido al riesgo de haber sido producido por un perro infectado con el virus de la rabia. La valoración de las medidas a tomar ha de ser determinada por el personal de salud que atienda la consulta.

En cuanto a la temporalidad de ocurrencia de los accidentes, estos predominaron entre los meses de diciembre a febrero, coincidente con el verano de Lima. Al respecto, Palacio et al. (2005) mencionan que debido a las elevadas temperaturas veraniegas, se puede observar en las calles una mayor afluencia de personas y canes sin control, con lo que la exposición a una mordedura aumenta. Chacma (2016) también encontró similares resultados en un estudio realizado en el Centro de Salud Playa Rímac ubicado en el Callao, y Eizaguirre (2016) que obtuvo mayor frecuencia de accidentes por canes durante marzo y abril, y de agosto a diciembre, meses calurosos en la ciudad de Arequipa.

Una de las variables de interés reportada en el estudio fue la ubicación anatómica de las mordeduras, la misma que influye sobre la decisión de realizar 
un protocolo de tratamiento post-exposición $\mathrm{y}$ la evaluación del uso de vacunación antirrábica únicamente o vacunación más suero hiperinmune antirrábico. El estudio reporta una mayor frecuencia de casos de mordedura con lesiones únicas en miembros inferiores, lo que estaría relacionado al predominio de casos en el grupo etario adulto, que por talla dejan principalmente expuestos los miembros inferiores, especialmente cuando se produce un ataque sorpresivo en vía pública. Cubillos (2014), en los servicios de salud de la Comuna de Quinta Normal en Santiago de Chile; Arroyo et al. (2015), a partir de encuestas en instituciones educativas en Huaraz - Ancash; Eizaguirre (2016), en el Hospital III Goyeneche en Arequipa y Gallegos et al (2018), con información de la Red de Salud en el distrito de Puente Piedra en Lima -Perú; también reportaron predominio de mordeduras en miembros inferiores.

Las lesiones en miembros superiores suelen predominar en ataques intradomiciliarios ya que cuando el can es conocido, la persona no se encuentra en estado de alerta. En niños también se presentan resultados similares, debido a sus características físicas que los hace más vulnerables. Así, Morales et al. (2011) en un estudio realizado en el Instituto Nacional de Salud del Niño, reportó predominio de lesiones en miembros superiores, miembros que utilizan para detener el ataque o para protegerse de las mordeduras en cara, cabeza y cuello.

La clasificación de los accidentes como provocados y no provocados suele depender del escenario de la mordedura. Sin embargo, el criterio de provocado puede darse por conductas que el humano no considera que sean un estímulo para un ataque, pero que el can puede tomarlo como una provocación (Palacio et al., 2005). Arroyo et al. (2015) y Talavera et al. (2018) también encontraron predominio en los ataques sorpresivos en sus estudios. Sin embargo, en estudios realizados en niños como el de Schvartzman y Pacín (2005) y Morales et al. (2011) predominaron los ataques provocados ya que los niños son menos conscientes de sus actos, actitudes que los animales lo perciben como amenaza o les causa molestia.

Las lesiones leves fueron las que predominaron en el estudio. Según la Norma Técnica de Salud Para la Vigilancia, Prevención y Control de la Rabia Humana en el Perú, en ellas se considera a las mordeduras o arañazos ocasionados por animales sin síntomas o sospecha de rabia, que ocasionan lesión generalmente única, superficial y localizada en cualquier parte del cuerpo a excepción de cara, cabeza, cuello, genitales o pulpejos de dedos en manos y pies (MINSA, 2017).

La mayoría de los accidentes no requirieron la aplicación de la vacuna antirrábica dada su clasificación (lesiones leves, en miembros inferiores, nula o baja probabilidad de que el can tuviera rabia). La Norma Técnica de Salud Para la Vigilancia, Prevención y Control de la Rabia Humana en el Perú recomienda que la profilaxis post-exposición debe ser indicada en el caso de mordeduras profundas con presencia de sangrado, en zonas próximas al sistema nervioso central (cuello, cara y cabeza), o zonas con importante inervación como los genitales y pulpejos de los dedos (MINSA, 2017).

El reconocimiento del can mordedor es importante para definir las medidas preventivas a tomar con la víctima del accidente. En ese aspecto se presentó un sub registro en la condición del can mordedor. Solo el $47.4 \%$ del total de fichas trabajadas contaban con esta información. En el estudio predominaron los canes huidos por lo que el esquema completo de vacuna antirrábica humana se debió considerar como parte del tratamiento post-exposición. Entre los canes que se lograron registrar se encontró una variabilidad de edades. Sin embargo, Palacio et al. (2005) mencionan que sin importar la edad del agresor, cualquier can puede ser capaz de producir una mordedura, en tanto Schvartzman y Pacín (2005) manifiestan que la tendencia a atacar depende de diversos factores como el estado de salud del animal, experiencias previas, la conducta del agraviado, entre otros.

La información de la raza de los animales agresores fue limitada, aunque se recabó información de la envergadura o tamaño, predominando los animales medianos y grandes. Schvartzman y Pacín (2005) también encontraron en su estudio que la mayoría de canes agresores fueron medianos a grandes, al igual que Arroyo et al. (2015). El reconocimiento de las razas suele ser difícil debido a que la población afectada no suele tener el conocimiento correcto de las características que definen una raza en particular. Se puede describir un animal con las características de una raza en particular y al final no serlo, pudiendo estigmatizar algunas razas de canes, especialmente aquellas que son catalogadas como potencialmente peligrosas. Según la Resolución Ministerial N¹7762002-SA/DM del Ministerio de Salud, en este grupo se incluyen los canes de raza Pit Bull Terrier, Dogo 
Argentino, Fila Brasilero, Tosa Japonesa, Bul Mastiff, Doberman y Rottweiler, e inclusive los cruces de ellas (MINSA, 2002).

El reconocimiento de que el animal fue vacunado contra la rabia es importante para determinar la responsabilidad de los propietarios en el cuidado preventivo de la salud de sus canes, en relación a dicha enfermedad, y el interés en participar activamente con los programas de prevención de la rabia en zonas no endémicas.

Los resultados del estudio muestran que los accidentes por mordedura siguen representando un problema de importancia en la salud pública y que debe enfrentarse a través de un plan preventivo que se soporte en el cumplimiento de normas como la Ley que regula el Régimen Jurídico de Canes. Además, debe considerar la participación de diversas instituciones como los organismos de salud y educación, gobiernos locales y la comunidad en conjunto, con un fuerte componente comunicacional que permita sensibilizar a la población acerca de la importancia de reportar este tipo de accidentes debido a su potencial impacto sanitario, epidemiológico, psicológico y social. Esta sensibilización debe incluir la obligatoriedad de hacer vacunar anualmente a los canes contra la rabia a fin de evitar la reintroducción de dicha zoonosis a zonas libres y de esta forma evitar el riesgo de presentación de rabia humana.

\section{CONCLUSIONES}

El estudio acerca de accidentes por mordedura de canes atendidos en el hospital "Sergio E. Bernales" de Comas llega a las siguientes conclusiones:

La mayoría de los accidentados procedían del distrito de Comas, pero residentes de distritos colindantes también recurrieron al Hospital Sergio E. Bernales.

La mayoría de afectados fueron adultos (30-59 años) de sexo masculino.

Predominaron los accidentes sorpresivos, de carácter leve, ubicados en miembros inferiores y con búsqueda de atención médica al día siguiente.

La condición de huidos fue la característica predominante entre animales mordedores. Entre los observados, predominaron los machos, de tamaño mediano y de 3 a 5 años de edad.

\section{Correspondencia}

Ivanna Door Matos

Correo electrónico: ivanna.door.m@upch.pe

\section{REFERENCIAS BIBLIOGRAFICAS}

1. Arroyo, V., Julca, G., Morales, D., \& León, D. (2015). Accidentes por mordedura de canes en estudiantes de instituciones educativas de la ciudad de Huaraz, Ancash, Perú. Revista Saludy Tecnología Veterinaria, 3(1), 1-9.

2. Chacma, D. (2016). Características epidemiológicas y clínicas de las mordeduras caninas. Centro de Salud Playa Rímac - Callao Enero 2011 - Diciembre 2015. [Tesis de pregrado]. Universidad de San Martín de Porres.

3. Ley $\mathrm{N}^{\circ} 27596$. Ley que regula el régimen jurídico de canes. (14 de diciembre de 2001). http://busquedas. elperuano.com.pe/download/full/7SXeVAz2 Ket8qUh_jMAog

4. Cubillos, M. (2014). Caracterización epidemiológica de las mordeduras de perro a la población humana notificadas en servicios de salud de la comuna de Quinta Normal, Santiago, 2012. [Tesis de pregrado]. Universidad de Chile.

5. Eizaguirre, A. (2016). Características epidemiológicas de las personas agredidas por perros que acudieron al Hospital III Goyeneche en el año 2015. [Tesis de pregrado]. Universidad Nacional de San Agustín.

6. Gallegos, L., Sulca, G., León, D., \& Falcón, N. (2018). Características de los accidentes por mordedura de canes registrados en la Red de Salud Puente Piedra durante el año 2015. MV Revista de Ciencias Veterinarias, 34(1).

7. Instituto Nacional de Estadística e Informática. (2014). Una mirada a Lima Metropolitana. Instituto Nacional de Estadística e Informática https://www. inei.gob.pe/media/MenuRecursivo/publicaciones_ digitales/Est/Lib1168/libro.pdf

8. Instituto Nacional de Estadística e Informática. (2017). Perú: Crecimiento y distribución de la población, 2017. Censos Nacionales 2017: XII de población y VII de vivienda. Instituto Nacional de Estadística e Informática.

9. Resolución Ministerial No 1776-2002-SA/DM. (11 de noviembre del 2002).Consideran en la relación de razas dec anes potencialmente peligrosos al Pit Bull Terrier, Dogo Argentino, Fila Brasilero, Tosa Japonesa, Bul Mastiff, Doberman y Rotweller. Diario Oficial El Peruano.

10. NTS N $\mathrm{N}^{\mathrm{a}}$ 131-MINSA/2017/DGIESP. (2017). Norma Técnica de Salud para la Prevención y Control de la Rabia Humana en el Perú. Diario Oficial El Peruano. 
11. Morales, C., Falcón, N., Hernández, H., \& Fernández, C. (2011). Accidentes por mordedura canina, casos registrados en un hospital de niños de Lima, Perú 1995-2009. Revista Peruana de Medicina Experimental y Salud Pública, 28(4), 639-642.

12. Novoa, D., León, D., \& Falcón, N. (2017). Accidentes por mordedura de perros en escolares de instituciones educativas públicas y privadas de San Martin de Porras, Lima-Perú. Revista Salud y Tecnología Veterinaria, 5, 1-7.

13. Organización Mundial de la Salud (2018). Mordedura de Animales. Nota descriptiva N³73. Organización Mundial de la Salud. http://www.who.int/ mediacentre/factsheets/fs373/es/

14. Palacio, J., León, M., \& García-Belenguer, S. (2005). Aspectos epidemiológicos de las mordeduras caninas. Gaceta Sanitaria, 19(1), 50-58.
15. Saad, C., \& Martínez, M. (2014). Protocolo de Vigilancia en Salud Pública-Rabia en humanos, perros y gatos. Instituto Nacional de Salud. Colombia.

16. Stokstad, E. (2017). Inside the global campaign to get rid of rabies. Revista Science. https:/www. sciencemag.org/news/2017/01/inside-globalcampaign-get-rid-rabies

17. Schvartzman, S. D., \& Pacín, M. B. (2005). Lesiones por mordedura de perro en niños. Archivos argentinos de pediatría, 103(5), 389-395.

18. Talavera, M., Gamboa, B., Gonzales, J., Huanambal, C., León, D., \& Falcón, N. (2018) Accidentes por mordedura de canes y conocimiento de rabia urbana en pobladores de Madre de Dios y Puno, Perú, 2014. Revista de Investigaciones Veterinarias del Perú, 29(3), 1025-1035. 